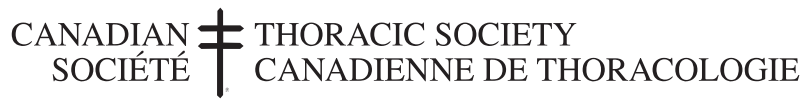

\section{The means to match our ambitions}

$\mathrm{M}$ ore than ever, the Canadian Thoracic Society (CTS) needs to align with its environment and leverage opportunities within it if it wants its impact as a specialty society in Canada to continue. Momentum has been achieved among the CTS, the Canadian Lung Association (CLA) and, to some extent, the American College of Chest Physicians (CHEST) and the pharmaceutical industry, to give the CTS a tangible plan, to ensure that its projects get the funding they need, and to ensure its sustainability.

Since 2007, CTS activities have been guided by a comprehensive strategic plan developed under the aegis of Dr Gordon Ford. Thanks to this foundational plan, we successfully carried out many activities and, working with our partners, accomplished a great deal with the implementation of the Canadian Respiratory Guidelines Committee, the Canadian Respiratory Conference, the National Respiratory Research Strategy and the Canadian Respiratory Research Network.

Nevertheless, the need to update our strategic plan and business model is clearly being felt. The future of the CTS is destined to play out in a new and more competitive environment characterized by increasing resource constraints. In this new environment, the Society must be able to continue to provide efficient and transparent management in order to contribute to an environment of trust for our various partners involved in respiratory health: researchers, clinicians, health professionals, government bodies, lung associations, pharmaceutical companies and others.

The Executive Committee and the CTS Board of Directors agreed to launch a strategic planning process during the summer that will lead us to a reaffirmed vision and will facilitate the process of choosing our priorities and allocating our resources. Thanks to this process, we can recast our ambitions and make sure we have the means to match them.

A steering committee will assist an external consulting firm (CapCOGITO) that has been retained for this important work. The committee will consist of Diane Lougheed (President-Elect), Debra Lynkowski (President and CEO of the Canadian Lung Association), Janet Sutherland (CTS Director) and me. Here are some of the many issues we will address:

- What should our core priorities be?

- What would the best management model be for us?

- How can we ensure the CTS is financially viable in the long term?

- What kind of organizational and governance structure would most likely lead to our success?

- What role should the CTS play in our relationship with the CLA?

- How can we ensure that our membership fees match the value and benefits of membership?

- What should we focus on to have the greatest possible impact on the field of respiratory health?

- Which partnerships should we maintain and strengthen?

Above all, the strategic planning process will enable us to restate our orientations, set our priorities for action and articulate our financial planning. It has become absolutely essential that the CTS's governance be efficient and uniting, that we maintain dialogue and strengthen our ties with partners like the CLA, and that we forge new partnerships. We must be resourceful as we face our greatest constraint: funding.

Any planning process must begin by taking stock of the situation and, especially, by being open to all ideas and suggestions, particularly

\section{Les moyens de nos ambitions}

T a Société canadienne de thoracologie (SCT) doit plus que jamais

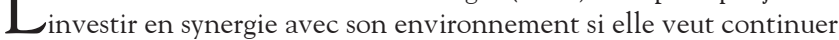
d'avoir un impact comme société professionnelle en santé respiratoire au Canada. On constate actuellement une effervescence entre la SCT, l'Association pulmonaire du Canada (APC) et, dans une certaine mesure, l'American College of Chest Physicians (CHEST) et l'industrie pharmaceutique, qui cherchent à doter la SCT d'un plan tangible, d'assurer le financement de ses projets, et à long terme, sa pérennité.

Depuis 2007, les activités de la SCT sont éclairées par le plan stratégique élaboré sous la tutelle de Gordon Ford. Grâce à ce plan, nous avons mené à bien nombre d'activités et connu un grand succès en collaboration avec nos partenaires, que ce soit la mise sur pied du Comité des lignes directrices canadiennes en santé respiratoire, du Congrès canadien sur la santé respiratoire, de la Stratégie nationale sur la recherche en santé respiratoire ou du Réseau canadien de recherche en santé respiratoire.

Néanmoins, le besoin de mettre à jour notre plan stratégique et notre modèle de gestion se fait sentir. L'avenir de la SCT se dessine dans un nouvel environnement, plus compétitif, aux ressources de plus en plus limitées. Dans cet environnement, la Société doit réussir à assurer une gestion efficiente et transparente, qui contribuera à un climat de confiance entre les différents partenaires en santé respiratoire (chercheurs, cliniciens, professionnels de la santé, organisations gouvernementales, associations pulmonaires, sociétés pharmaceutiques, entre autres).

Pendant l'été, le Conseil exécutif et le conseil d'administration de la SCT se sont entendus pour lancer une démarche de planification stratégique, en vue de réaffirmer notre vision et de faciliter la sélection des priorités et l'affectation des ressources. Ainsi, nous pourrons en quelque sorte réaffirmer nos ambitions et nous donner les moyens de les concrétiser.

Un comité de pilotage assistera le consultant externe (CapCOGITO) dont les services ont été retenus. Ce comité sera constitué des membres suivants : Diane Lougheed (présidente désignée), Debra Lynkowski (présidente-directrice générale de l'Association pulmonaire), Janet Sutherland (directrice de la SCT) et moi-même. Plusieurs questions seront abordées, notamment :

- Quelles seront nos grandes priorités?

- Quel serait le meilleur modèle de gestion à suivre?

- Comment assurer la viabilité financière de la SCT à long terme?

- Quelle structure organisationnelle et de gouvernance se prête le mieux à notre succès?

- Quel rôle la SCT doit-elle assumer auprès de l'APC?

- Comment s'assurer que la cotisation correspond à la valeur et aux avantages de l'adhésion?

- Où mettre l'accent pour avoir un impact maximal en santé respiratoire?

- Quels partenariats doit-on maintenir et renforcer?

Plus que tout, la démarche de planification stratégique permettra de réaffirmer nos orientations, d'établir nos priorités et de préparer une planification financière. Il devient absolument essentiel de doter la SCT d'une gouvernance efficace et rassembleuse, de maintenir le dialogue, de renforcer les liens avec des partenaires comme l'APC et de 
those coming from our members. In September, your Society called on you to respond to a survey to gather input on some of the questions noted above. I hope you took advantage of this opportunity to share your views. If you haven't yet done so, there's still time. Please share your ideas by e-mail at CTSstrategy@lung.ca.

Our organization, and our leading partners, the CLA and the Canadian Respiratory Health Professionals (CRHP), need you. I hope that you will answer our call in great numbers. Your involvement as a CTS member has never been so important and necessary.

Cordially,

Jean Bourbeau MD MSc FRCPC

President, Canadian Thoracic Society

nouer de nouveaux partenariats. Nous devons faire preuve de débrouillardise devant notre plus grande contrainte, le financement.

Dans toute démarche de planification, il faut d'abord faire l'état de la situation. Il faut également être à l'écoute, particulièrement des membres. Dans cet esprit, en septembre, nous vous avons sollicités pour répondre à une enquête par questionnaire. J'espère que vous en avez profité pour partager votre point de vue. Sinon, il n'est pas trop tard! Faites-nous part de vos idées par courriel, à strategieSCT@poumon.ca.

Avec ses partenaires privilégiés, l'APC et les Professionnels canadiens en santé respiratoire (PCSR), la SCT a besoin de vous. J'espère que vous répondrez en grand nombre à notre appel. Plus que jamais, votre participation comme membre de la SCT est nécessaire.

Salutations distinguées, Jean Bourbeau MD, M. Sc., FRCPC

Président, Société canadienne de thoracologie 


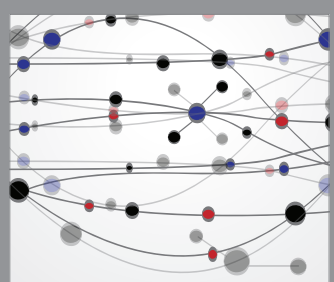

The Scientific World Journal
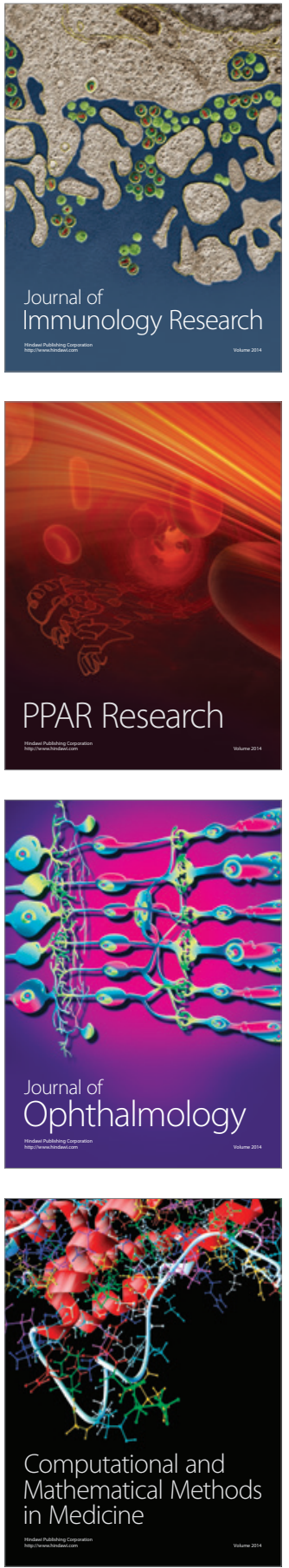

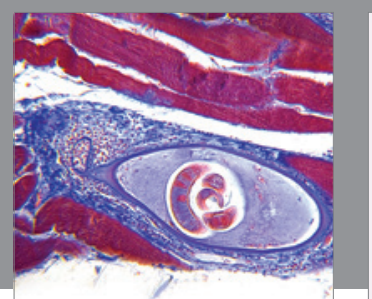

Gastroenterology Research and Practice

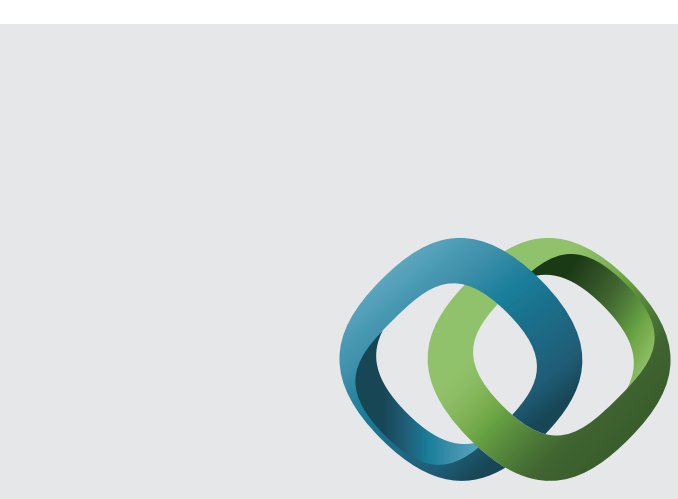

\section{Hindawi}

Submit your manuscripts at

http://www.hindawi.com
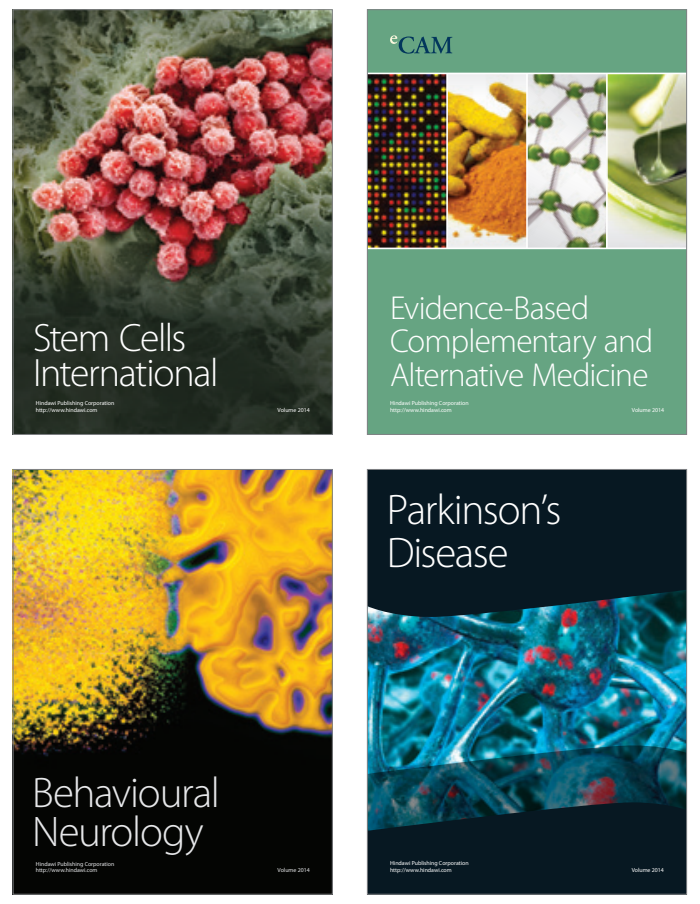
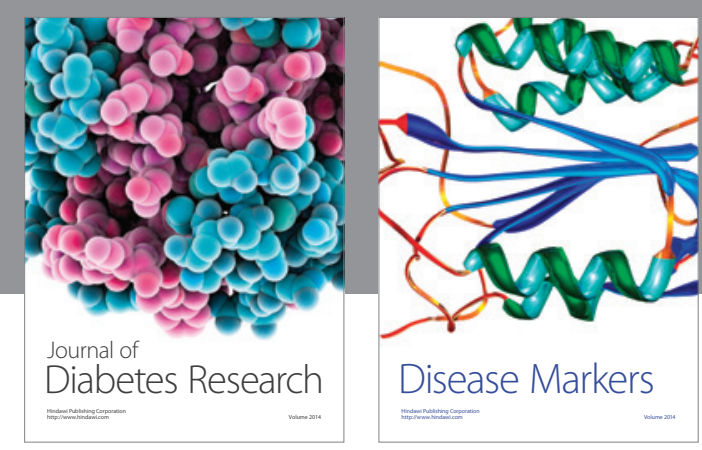

Disease Markers
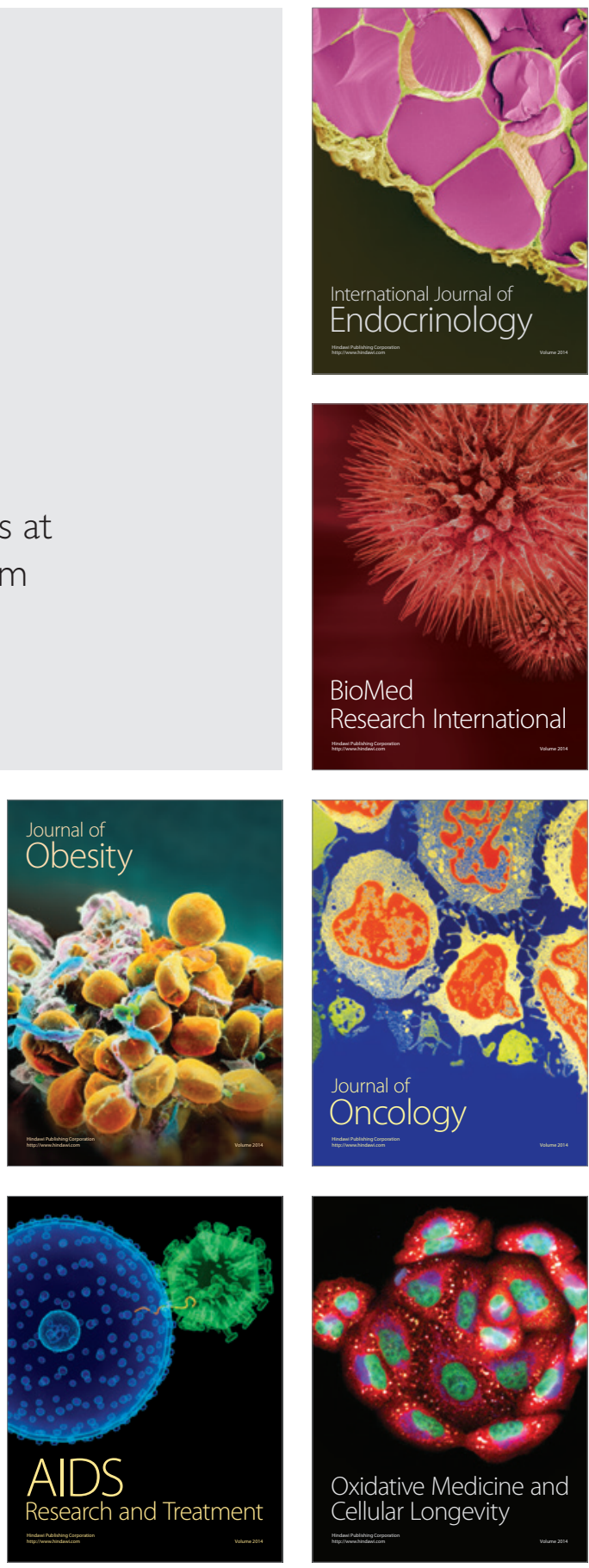